Article

\title{
Study of the Properties of a Hybrid Piezoelectric and Electromagnetic Energy Harvester for a Civil Engineering Low-Frequency Sloshing Environment
}

\author{
Nan Wu (D), Yuncheng He, Jiyang Fu * and Peng Liao
}

check for updates

Citation: Wu, N.; He, Y.; Fu, J.; Liao, P. Study of the Properties of a Hybrid Piezoelectric and Electromagnetic Energy Harvester for a Civil Engineering Low-Frequency Sloshing Environment. Energies 2021, 14, 391. https://doi.org/10.3390/en14020391

Received: 22 December 2020 Accepted: 9 January 2021 Published: 12 January 2021

Publisher's Note: MDPI stays neutral with regard to jurisdictional clai$\mathrm{ms}$ in published maps and institutional affiliations.

Copyright: (C) 2021 by the authors. Licensee MDPI, Basel, Switzerland. This article is an open access article distributed under the terms and conditions of the Creative Commons Attribution (CC BY) license (https:// creativecommons.org/licenses/by/ $4.0 /)$.
Guangzhou University-Tamkang University Joint Research Centre for Engineering Structure Disaster Prevention and Control, Guangzhou University, Guangzhou 510006, China; wunan@gzhu.edu.cn (N.W.); yuncheng@gzhu.edu.cn (Y.H.); liaopeng650@foxmail.com (P.L.)

* Correspondence: jiyangfu@gzhu.edu.cn

\begin{abstract}
In this paper a novel hybrid piezoelectric and electromagnetic energy harvester for civil engineering low-frequency sloshing environment is reported. The architecture, fabrication and characterization of the harvester are discussed. The hybrid energy harvester is composed of a permanent magnet, copper coil, and PVDF(polyvinylidene difluoride) piezoelectric film, and the upper U-tube device containing a cylindrical fluid barrier is connected to the foundation support plate by a hinge and spring. The two primary means of energy collection were through the vortex street, which alternately impacted the PVDF piezoelectric film through fluid shedding, and the electromotive force (EMF) induced by changes in the magnetic field position in the conducting coil. Experimentally, the maximum output power of the piezoelectric transformer of the hybrid energy harvester was $2.47 \mu \mathrm{W}$ (circuit load $270 \mathrm{k} \Omega$; liquid level height $80 \mathrm{~mm}$ ); and the maximum output power of the electromagnetic generator was $2.72 \mu \mathrm{W}$ (circuit load $470 \mathrm{k} \Omega$; liquid level height $60 \mathrm{~mm}$ ). The low-frequency sloshing energy collected by this energy harvester can drive microsensors for civil engineering monitoring.
\end{abstract}

Keywords: energy harvester; piezoelectricity; low frequency; civil engineering

\section{Introduction}

Piezoelectric and magnetoelectric energy-harvesting devices based on mechanical vibration have attracted considerable attention among several alternatives to conventional batteries, as they provide stable and continuous power supplies for microsensors because of the abundance of vibration sources in nature [1]. For some civil engineering structure monitoring networks deployed in harsh environments, routine maintenance consumes large amounts of resources. Because of environmental concerns, structural location and engineering design, the replacement and charging of batteries can be impossible. Therefore, the development of new ways to supply energy is important for overcoming the limitations of traditional batteries [2,3].

Previous research on piezoelectric energy harvesters has focused on improving the power generation efficiency of piezoelectric devices through amplitude/frequency amplification technology, resonance tuning technology and nonlinear technology [4-6]. Chen and Jiang (2015) designed and tested a nonlinear energy harvester using the internal resonance method in nonlinear dynamics theory [7]. Li and Qin (2015) studied the vibration energy acquisition properties of piezoelectric laminated beams in the buckling state [8]. Cao et al. (2015) studied the fractional damping energy acquisition system. Experimental research has shown that the magnetoelectric piezoelectric coupling energy harvester has a wide bandwidth under low-frequency excitation [9-12]. Zhou et al. (2014) studied the nonlinear response characteristics of the tri-stable energy collector and showed that multi-steady state technology can effectively broaden the absorption bandwidth of the energy collector [13]. 
In addition, Yao et al. (2015) and Li et al. (2015) studied the dynamic characteristics and the working efficiency of the bistable vibration energy harvester using theoretical and experimental methods, respectively [14].

However, the design frequency of the energy harvester is relatively high, and the resonant frequency of many devices has been designed to remain at the level of $100 \mathrm{~Hz}$, which severely limits applicability [15-17]. In civil engineering, low frequency and small amplitude are fundamental for load vibration in buildings, bridges and other structures. Cahill et al. used six sets of piezoelectric cantilever beams made of aluminum plate and PVDF(polyvinylidene difluoride) to form a system showing broadband energy collection, which was arranged under a full-scale bridge [18]. The natural frequencies of six groups of piezoelectric cantilever beams ranged from $6.2 \mathrm{~Hz}$ to $20.6 \mathrm{~Hz}$ and can be used to monitor the vibration frequency of the bridge structure as vehicles pass. When the input frequency and acceleration are $4.1 \mathrm{~Hz}$ and $0.02 \mathrm{~g}$, respectively, the reported harvester delivered a maximum power of $3.43 \mathrm{~mW}$ to a load resistance of $145 \Omega$. Furthermore, a low-cost, temperature-resistant and waterproof 3-axis bridge condition monitoring WSN (Wireless Sensor Networks) was developed by Neitzel et al. (2011), and the wireless sensor node can continuously monitor and record data using the bridge's vibrations as a power source $[19,20]$. A new energy harvester that can harvest traffic-induced bridge vibrations was described by Lee et al. (2012), and the fabricated harvester can generate an electrical power of $2 \mu \mathrm{W}$ at a frequency range of $1-4 \mathrm{~Hz}[21,22]$.

In addition, hybrid (piezoelectric electromagnetic) energy harvesting technology has recently emerged and has received increased research attention [23,24]. Hybrid energy harvesting technology has multiple resonant frequencies, wide working band width and a dual conversion mechanism, which permits the generation of more energy. Challa et al. (2015) developed a cantilever beam hybrid energy harvester, and the power of this harvester was increased by $30 \%$ compared with the single piezoelectric or electromagnetic module [25]. Toyabur et al. (2015) proposed a multi-mode hybrid energy harvester that greatly increased the applicable frequency of the device, and the resonance frequencies of the device were 12, 15, 17 and $22 \mathrm{~Hz}$ [26]. Iqbal et al. (2015) designed a new multimodal hybrid bridge energy harvester using a combined piezoelectric and electromagnetic conversion process that operates in three low-frequency resonant modes $(12.5,34$ and $45 \mathrm{~Hz})$ and produces a maximum power of $2214.32 \mu \mathrm{W}$ [27].

Here, we developed an energy-harvesting device that converts the low-frequency sloshing in a civil engineering environment into liquid flow, thereby collecting the vibration energy in the environment and converting it into useful electrical energy. The harvester is designed to use a combination of piezoelectric and electromagnetic conversion rather than a single energy collection technology. The combination of piezoelectric and electromagnetic energy harvesting can effectively widen the low-frequency working range of the device and improve the energy collection efficiency of the vibration environment.

\section{Design and Working Principle}

\subsection{Design}

The schematic of the proposed hybrid energy harvester for harvesting mechanical excitations is shown in Figure 1a. It consists of a square U-tube device in the upper part, and a base and an electromagnetic energy collecting device in the lower part; epoxy resin is the main material of the upper parts. In order to make the fluid in the square tube produce more impact and disturbance on the side wall, two bluff body (epoxy resin with a diameter of $20 \mathrm{~mm}$ ) are designed in the horizontal pipe fitting at the bottom of the U-tube, and there is a rectangular area $(20 \times 200 \mathrm{~mm})$ on both sides of this pipe, which is replaced by a butyl rubber film. Fluids of different heights $(\mathrm{h})$ are added to match the vibration frequencies of the device to the external environment. When the device is excited by external shaking, the U-tube device rotates back and forth in the vertical plane. As shown in Figure 1b, the flow of liquid in the pipe produces a Karman (Kármán) vortex street behind the bluff body; thus, the shedding of the vortex impacts the wall of the butyl rubber 
material, and part of the vibration energy in the environment is converted into electrical energy by the PVDF piezoelectric film tightly attached to it. Meanwhile, the magnetic field position on both sides of the U-tube bottom changes, the two coils on both sides of the base experience constant magnetic flux density, and an electromotive force (emf) is induced in the conductive coil according to Faraday's law of electromagnetic induction.

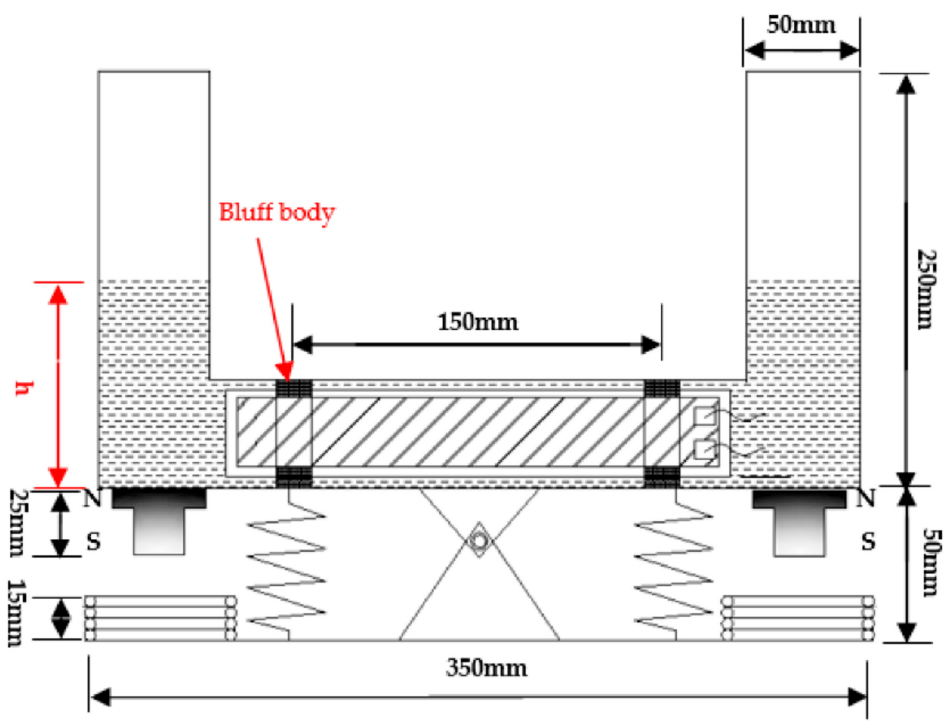

(a)

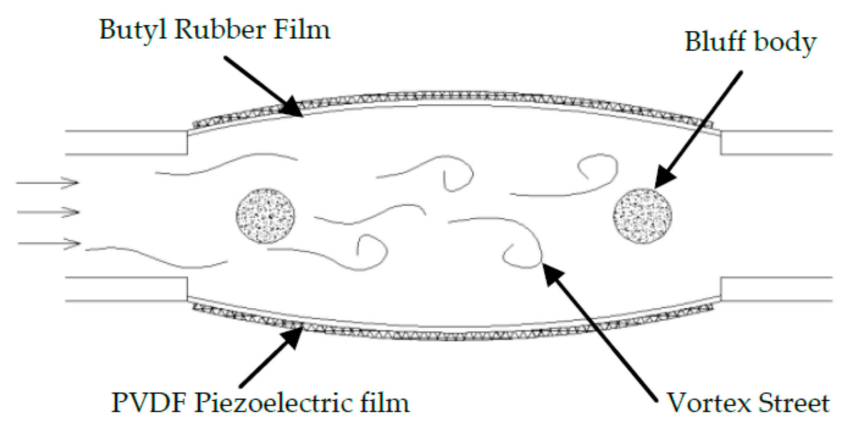

(b)

Figure 1. The hybrid energy harvester's architecture: (a) Diagram of the composition structure; (b) Schematic diagram of the vortex street.

\subsection{Modeling of Prototypes}

The energy equation of fluid is the following:

$$
Z_{1}+\frac{p_{1}}{\rho g}+\frac{u_{1}^{2}}{2 g}=Z_{2}+\frac{p_{2}}{\rho g}+\frac{u_{2}^{2}}{2 g}+h_{\omega}^{\prime}
$$

where $Z_{1}$ and $Z_{2}$ are the position heads (equivalent to different $h$ values in this study), $p_{1}$ and $p_{2}$ are the pressure heads, and $h_{\omega}$ is head loss. When the device is excited by ambient vibration, the U-tube rotates, resulting in a height difference between the liquid levels of the two pipe walls, and the velocity of the liquid (i.e., when the height difference between the two sides of the liquid level is $\mathrm{h}$ ) in the U-tube is $u=\sqrt{2 g h}$. At the same time, when the fluid passes through the blocking fluid (cylinder) at the bottom of the U-tube, an unsteady 
wake is produced, causing periodic change in the pressure on both sides of the pipe wall. When the velocity is in the sub-critical range $\left(3 \times 10^{2}<\operatorname{Re}<3 \times 10^{5}, \mathrm{R}_{e}=\frac{\rho V L}{\mu}\right.$, where $\rho$ and $\mu$ are fluid density and dynamic viscosity coefficient, respectively, and $\mathrm{V}$ and $\mathrm{L}$ are characteristic velocity and characteristic length of the flow field, respectively), the Karman vortex street appears.

Low-frequency vibration is the main characteristic of civil engineering structure vibration. The natural frequency of the device is designed to meet the application environment of civil engineering. The internal energy of the liquid remains unchanged in the process of motion. The motion equation of the liquid is established by the Lagrange equation as follows [28,29]:

$$
\frac{d}{d t}\left[\frac{\partial(T-U)}{\partial \dot{x}_{T}}\right]-\frac{\partial(T-U)}{\partial x_{T}}=Q
$$

The kinetic energy of the liquid oscillation is:

$$
T=\int_{V_{1}} \frac{1}{2} \dot{x}_{T}^{2} \rho d V+\int_{V_{2}} \frac{1}{2} \rho\left[\dot{x}_{T}+\dot{x}_{S}\right] d V+\int_{V_{3}} \frac{1}{2} \dot{x}_{T}^{2} \rho d V
$$

The potential energy of the liquid is:

$$
U=\rho g A \int_{0}^{H-x_{T}} z d z+\rho g A \int_{0}^{H+x_{T}} z d z+\frac{1}{2} k_{T 2} x_{T}^{2}
$$

The head loss of the liquid passing through the blocking fluid (cylinder) at the bottom of the U-tube is as follows:

$$
Q=-(\rho g A)\left(\frac{\xi\left|\dot{x}_{T}\right| \dot{x}_{T}}{2 g}\right)
$$

In conclusion, the motion equation (6) and natural frequency (7) of liquid are obtained

$$
\begin{gathered}
\rho A L \ddot{x}_{T}+\frac{1}{2} \rho A \xi\left|\dot{x}_{T}\right| \dot{x}_{T}+2 \rho A g x_{T}+k_{T 2} x_{T}=-\rho A B \ddot{x}_{S} \\
\omega_{T}=\sqrt{\frac{2 \rho A g+k_{T 2}}{\rho A L}}
\end{gathered}
$$

where $x_{T}$ is the distance from the equilibrium position of a liquid during vibration; $T$ and $U$ represent the kinetic energy and potential energy of the liquid in motion, respectively; $A$ is the cross-sectional area of the U-tube; $\rho$ is the liquid density; $h$ is the height of the static liquid level; $B$ is the center distance between the two vertical pipes; $x_{s}$ is the displacement caused by environmental vibration; $k_{T 2}$ is the stiffness of the spring; $g$ is the acceleration of gravity; $V$ is the vertical part of the U-tube; and $z$ is the distance between the integral unit and the horizontal pipe axis.

According to the above theoretical analysis, the natural frequencies of the device at different liquid level heights were calculated (Table 1).

Table 1. The harvester and experimental condition parameters.

\begin{tabular}{ccccccc}
\hline $\begin{array}{c}\text { Project } \\
\text { Number }\end{array}$ & $\begin{array}{c}\text { Excitation } \\
\text { Waveform }\end{array}$ & $\begin{array}{c}\text { Liquid Level } \\
\text { Height } \\
\text { (mm) }\end{array}$ & $\begin{array}{c}\text { Natural Frequency } \\
\text { (rad/s) }\end{array}$ & $\begin{array}{c}\text { Vibration Period } \\
\text { (s) }\end{array}$ & $\begin{array}{c}\text { Vibration } \\
\text { Frequency } \\
\text { (Hz) }\end{array}$ & $\begin{array}{c}\text { Amplitude } \\
\text { (mm) }\end{array}$ \\
\hline H-40 & sine wave & 40 & 7.85 & 1.25 & 0.80 & 25 \\
H-60 & sine wave & 60 & 7.68 & 1.22 & 0.82 & 25 \\
H-80 & sine wave & 80 & 7.36 & 1.17 & 0.85 & 25 \\
H-100 & sine wave & 100 & 7.02 & 1.12 & 0.89 & 25 \\
H-120 & sine wave & 120 & 6.81 & 1.08 & 0.93 & 25 \\
\hline
\end{tabular}




\section{Prototype Fabrication and Experimental Setup}

A macroscale prototype was fabricated for the in-lab verification of the proposed hybrid energy harvester (Figure 2). The bottom base, support and upper U-tube structure are composed of 3-mm thick PMMA (Polymeric Methyl Methacrylate), and the detailed dimensions are shown in Figure 1a. There was a rectangular area $(200 \times 30 \mathrm{~mm})$ on both sides of the horizontal pipe fitting at the bottom of the U-tube structure, which was replaced by a butyl rubber film, and the bonding of the two materials was treated to prevent water leakage by the high molecular waterproof membrane. The PVDF piezoelectric film (manufactured by Zhi-Mei-Kang Technology (Shenzhen, China) Co., Ltd.; the size and type were $170 \times 20 \mathrm{~mm}$ and IPS-17020) was bonded to butyl adhesive film by cyanoacrylate glue. The bottom of both sides of the U-tube structure held a Neodymium (NdFeB) permanent cylindrical magnet of two sizes (the upper part of the magnet was a cylinder with a diameter of $28 \mathrm{~mm}$ and a height of $5 \mathrm{~mm}$, and the lower part was a cylinder of $15 \mathrm{~mm}$ and a height of $20 \mathrm{~mm}$; the magnetic flux density was $0.9 \mathrm{~T}$ ). Underneath was a 20-mm diameter copper wire coil (inductance $2.1 \mathrm{mh}$ ). Two springs (made of 65-Mn spring steel, wire diameter $1 \mathrm{~mm}$, outer diameter $20 \mathrm{~mm}$, height $50 \mathrm{~mm}$, stiffness $0.21 \mathrm{~N} / \mathrm{mm}$ ) were distributed beside the coil to make the upper U-tube structure rotate back and forth.

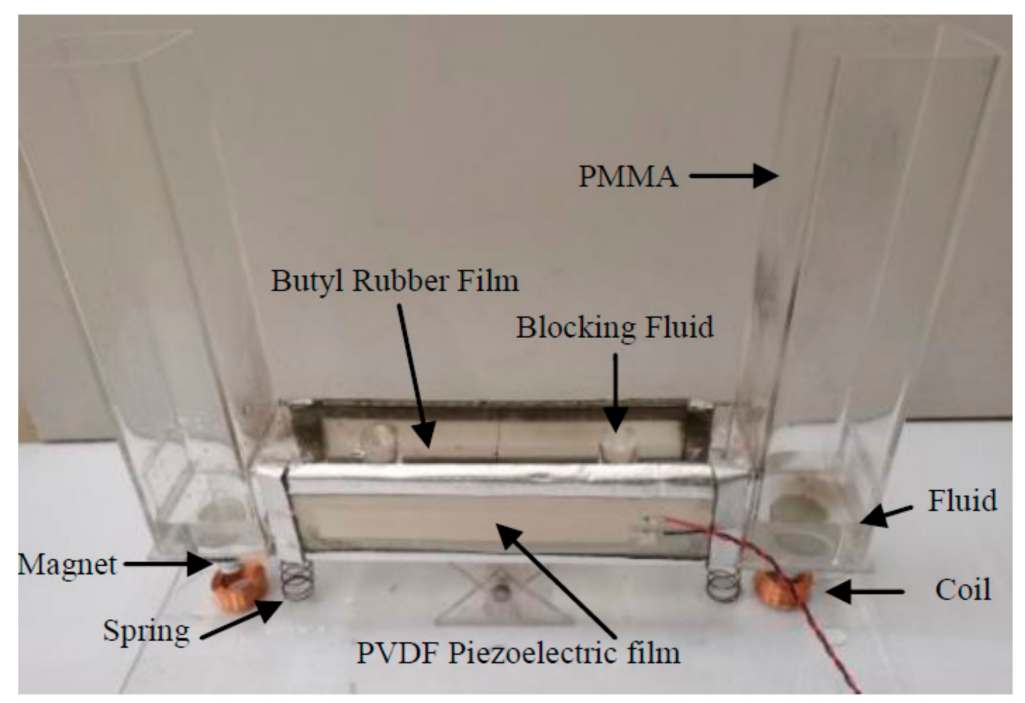

Figure 2. Photograph of the fabricated hybrid energy harvester.

According to the theoretical analysis of the hybrid energy harvester, the natural vibration frequency $(0.8-0.93 \mathrm{~Hz})$ of the device was obtained. Different liquid level heights affect the natural vibration frequency of the device; thus, it can be adjusted according to different external environments to produce the optimal environmental energy acquisition effect.

The experimental setup to test the fabricated prototype is the shaking table shown in Figure 3. The shaking table was derived by an electric motor manufactured by ACS Motion Control Company in USA, and its motion control was controlled by an ACS SPiiPlus ADK control system. The shaking table can generate modulation frequencies of $0.2 \sim 500 \mathrm{~Hz}$ in the horizontal direction ( $Y$ direction), with amplitudes of 0-100 $\mathrm{mm}$ and a maximum acceleration of $10 \mathrm{~g}$. DH8304 high-performance dynamic signal test and analysis system (manufactured by Jiangsu Donghua Testing Technology Co., Ltd., 208 Xingang Avenue, Jiangsu Province, China) was used for voltage signal acquisition and analysis.

The hybrid energy acquisition device was tested using the aforementioned equipment. At each liquid level, the shaking table was designed to apply an external excitation load with the same natural frequency as the device, and the output voltage of the piezoelectric and electromagnetic parts under the open circuit and different load resistances were tested, respectively, to evaluate the energy acquisition of the device (Figures 4-6 and Table 1). 


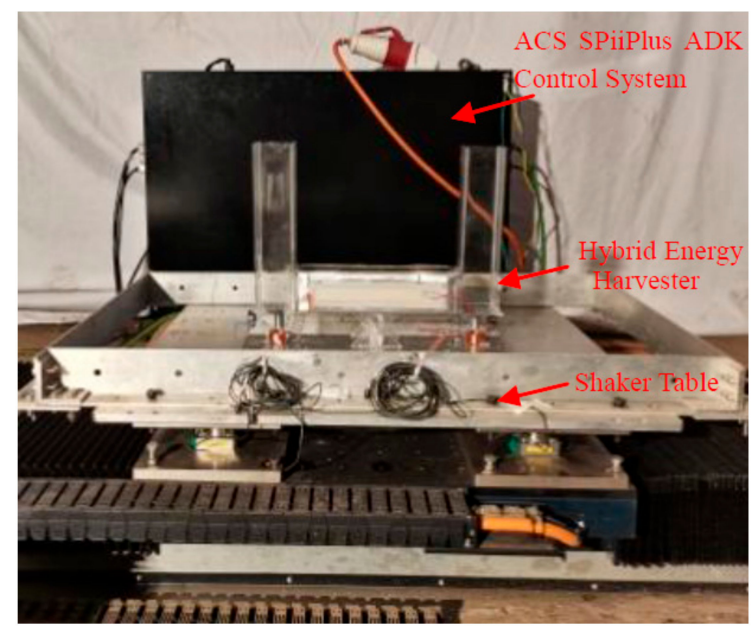

Figure 3. Experimental setup for the hybrid energy harvester.

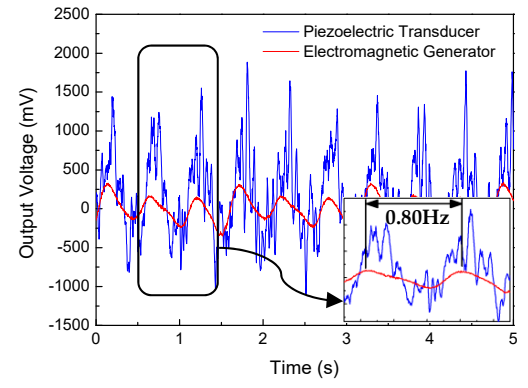

(a)

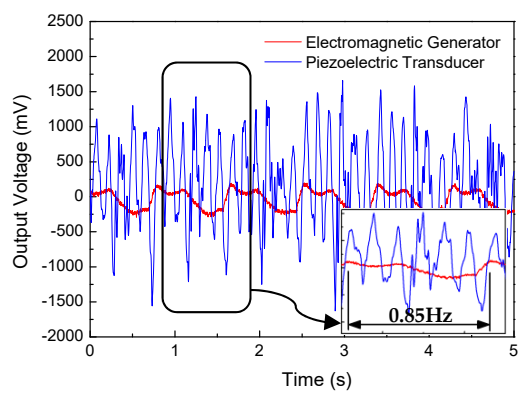

(c)

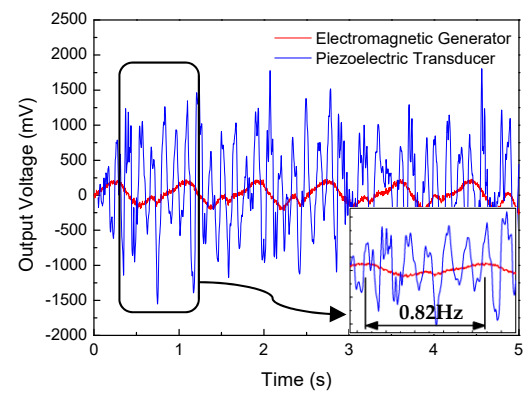

(b)

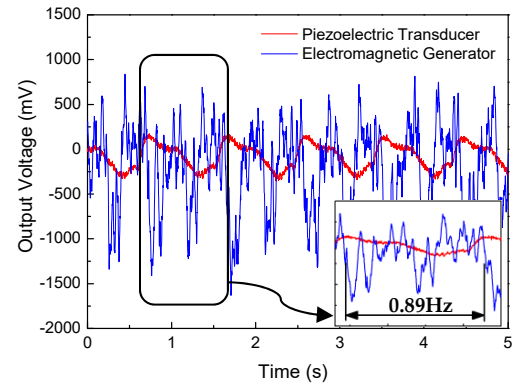

(d)

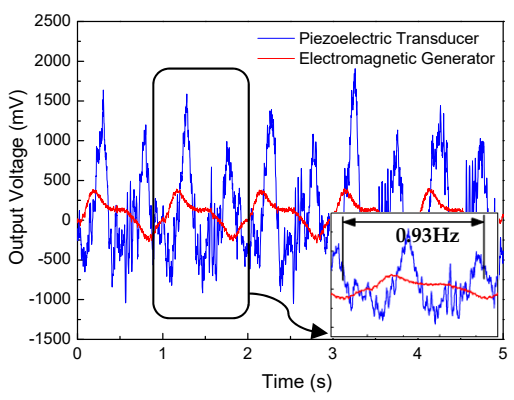

(e)

Figure 4. Output voltage signals of the hybrid energy harvester with different liquid levels: $(\mathbf{a}) \mathrm{h}=$ $40 \mathrm{~mm}$; (b) h = $60 \mathrm{~mm}$; (c) h = $80 \mathrm{~mm} ;$ (d) h = $100 \mathrm{~mm}$; and (e) h=120 mm. 


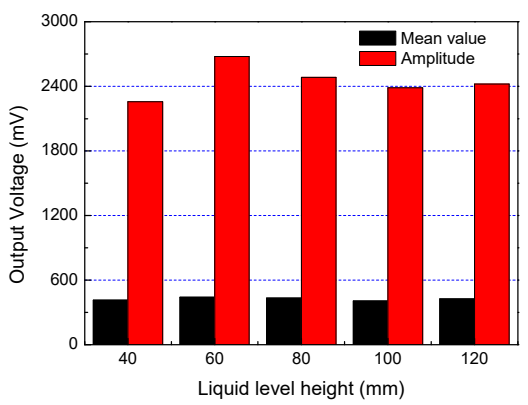

(a)

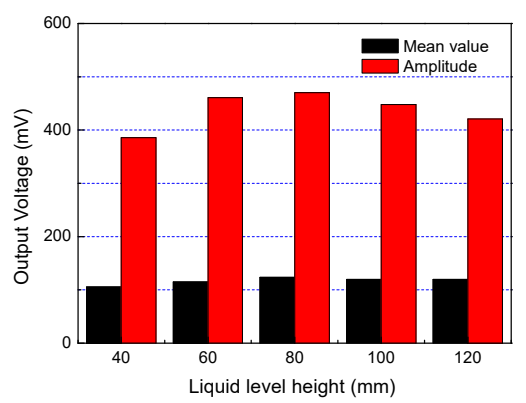

(b)

Figure 5. Bar graph of the hybrid energy harvester output voltage information: (a) Piezoelectric transducer; (b) Electromagnetic generator.

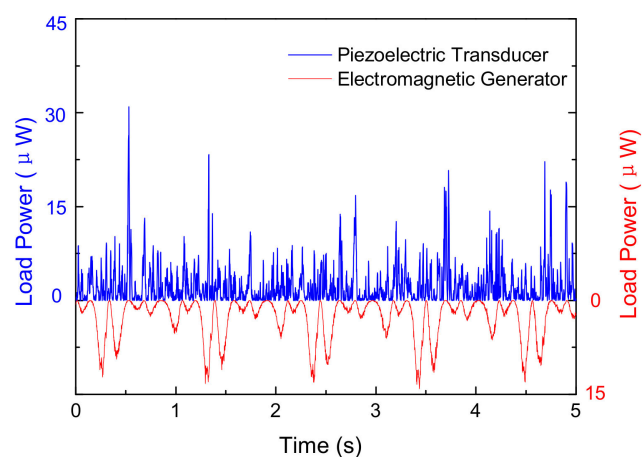

(a)

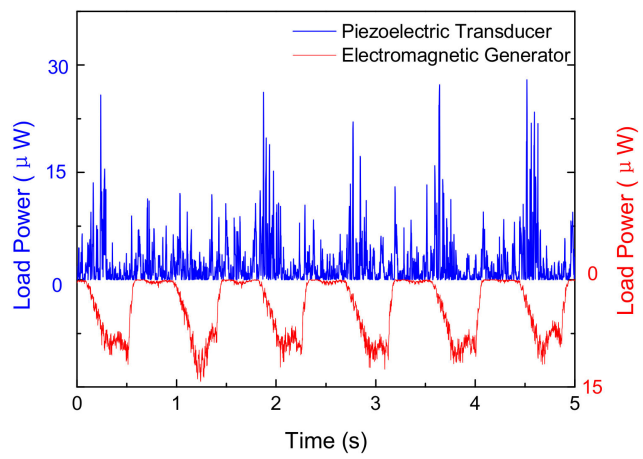

(c)

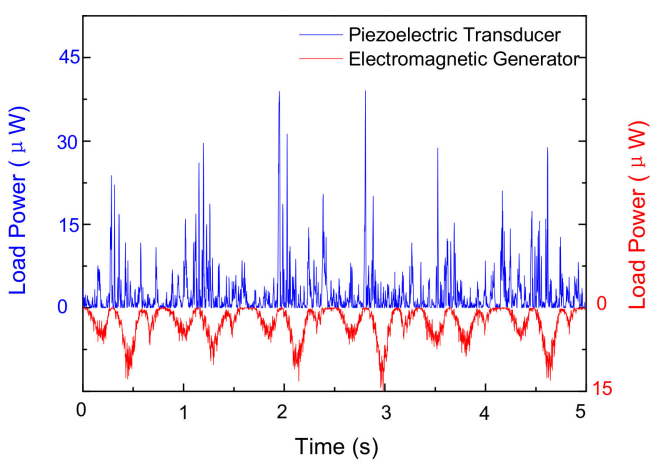

(b)

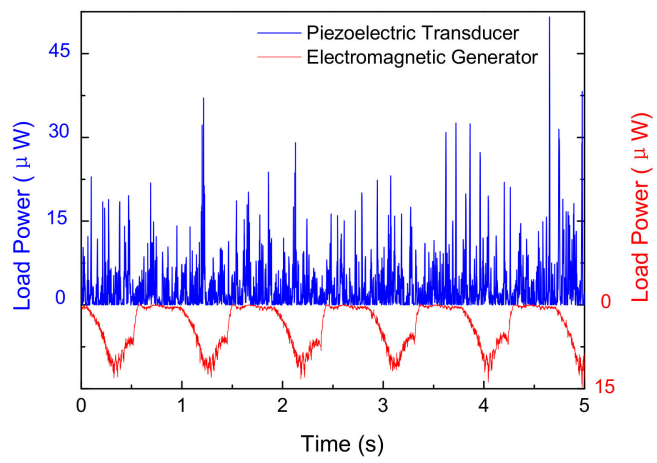

(d)

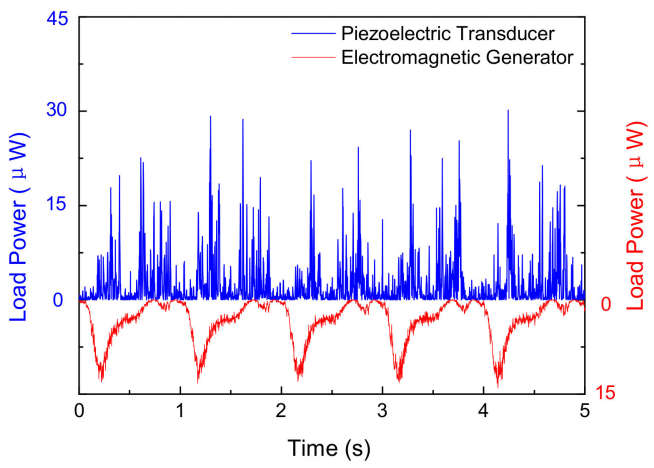

(e)

Figure 6. Output power signals (Load resistance is $270 \mathrm{k} \Omega$ ) of the hybrid energy harvester at different liquid levels: (a) $\mathrm{h}=40 \mathrm{~mm}$; (b) h $=60 \mathrm{~mm}$; (c) h $=80 \mathrm{~mm}$; (d) h $=100 \mathrm{~mm}$; and (e) h = $120 \mathrm{~mm}$. 


\section{Prototype Fabrication and Experimental Setup}

\subsection{Characterization of the Open-Circuit Voltage of the Hybrid Energy Harvester}

Five types of test conditions with different liquid level heights were used for the hybrid energy harvester, and their natural vibration frequencies were calculated and applied to the device through the shaking table test. The open-circuit output voltage signals (piezoelectric and electrical magnetic ports) of the device when resonance occurred at different liquid level heights were obtained (Figure 3 and Table 1). As the liquid level height increased from $40 \mathrm{~mm}$ to $120 \mathrm{~mm}$, the natural vibration frequency of the device decreased from $7.85 \mathrm{rad} / \mathrm{s}$ (the liquid level height is $40 \mathrm{~mm}$ ) to $6.81 \mathrm{rad} / \mathrm{s}$ (the liquid level height is $120 \mathrm{~mm}$ ), a decrease of $13.25 \%$; thus, the excitation frequency gradually increased from $0.80 \mathrm{~Hz}(40-\mathrm{mm}$ liquid level) to $0.93 \mathrm{~Hz}$ (120-mm liquid level).

As the liquid level increased from $40 \mathrm{~mm}$ to $120 \mathrm{~mm}$, the maximum voltage amplitude of the piezoelectric device was $2677.31 \mathrm{mV}$ (the liquid level height was $60 \mathrm{~mm}$ ), the minimum was $2257.26 \mathrm{mV}$ (the liquid level height was $40 \mathrm{~mm}$ ), and the variation amplitude was $15.7 \%$. The maximum average voltage output value was $441.89 \mathrm{mV}$ (the liquid level height was $60 \mathrm{~mm}$ ), the minimum was $406.30 \mathrm{mV}$ (the liquid level height was $100 \mathrm{~mm}$ ), and the variation amplitude was $8.1 \%$. When the external excitation causes resonance at different liquid level heights in the electromagnetic generator, the open circuit output voltage signal reaches its optimal value when the liquid level is $80 \mathrm{~mm}$, with an amplitude of $470.61 \mathrm{mV}$ and an average value of $123.73 \mathrm{mV}$. When the liquid level height was $40 \mathrm{~mm}$, the amplitude $(386.01 \mathrm{mV})$ and the mean value $(105.78 \mathrm{mV})$ were the lowest.

The change in the proportion of the amplitude was $17.98 \%$, and that of the mean value was $14.50 \%$ (Figure 5 ).

\subsection{Characterization of the Power of the Hybrid Energy Harvester}

To explore the generating power of the hybrid energy harvester under different load conditions, seven different resistors $(1 \sim 470 \mathrm{k} \Omega)$ were used to form a closed-circuit cycle, and the output power of the hybrid energy harvester in which resonance occurs at different liquid level heights was obtained. The results are shown in Tables 2 and 3. Figures 6 and 7 show the output power signals of the piezoelectric transformer and the electromagnetic generator with a resistance value of $270 \mathrm{k} \Omega$.

Table 2. Power of the piezoelectric transducer under different conditions.

\begin{tabular}{cccccc}
\hline $\begin{array}{c}\text { Liquid Level Heigh(mm) } \\
\text { Load Resistance }(\mathbf{k} \boldsymbol{\Omega})\end{array}$ & $\mathbf{4 0}$ & $\mathbf{6 0}$ & $\mathbf{8 0}$ & $\mathbf{1 0 0}$ & $\mathbf{1 2 0}$ \\
\cline { 1 - 5 } 470 & 1.91 & 2.10 & 2.19 & 1.99 & 1.95 \\
390 & 2.00 & 2.28 & 2.30 & 2.09 & 2.05 \\
270 & 2.12 & 2.46 & 2.47 & 2.28 & 2.24 \\
150 & 1.81 & 2.03 & 2.11 & 1.99 & 1.93 \\
51 & 1.06 & 1.31 & 1.36 & 1.22 & 1.19 \\
10 & 0.78 & 1.05 & 1.11 & 0.91 & 0.89 \\
1 & 0.69 & 1.01 & 1.05 & 0.89 & 0.80 \\
\hline
\end{tabular}

Table 3. Power of the electromagnetic generator under different conditions.

\begin{tabular}{cccccc}
\hline $\begin{array}{c}\text { Liquid Level Heigh }(\mathbf{m m}) \\
\text { Load Resistance }(\mathbf{k} \Omega)\end{array}$ & $\mathbf{4 0}$ & $\mathbf{6 0}$ & $\mathbf{8 0}$ & $\mathbf{1 0 0}$ & $\mathbf{1 2 0}$ \\
\hline 470 & 2.63 & 2.72 & 2.65 & 2.50 & 2.49 \\
390 & 2.61 & 2.70 & 2.64 & 2.47 & 2.46 \\
270 & 2.60 & 2.70 & 2.68 & 2.49 & 2.47 \\
150 & 2.63 & 2.69 & 2.66 & 2.53 & 2.50 \\
51 & 2.61 & 2.72 & 2.68 & 2.51 & 2.46 \\
10 & 2.60 & 2.70 & 2.66 & 2.52 & 2.47 \\
1 & 2.58 & 2.71 & 2.63 & 2.50 & 2.45 \\
\hline
\end{tabular}




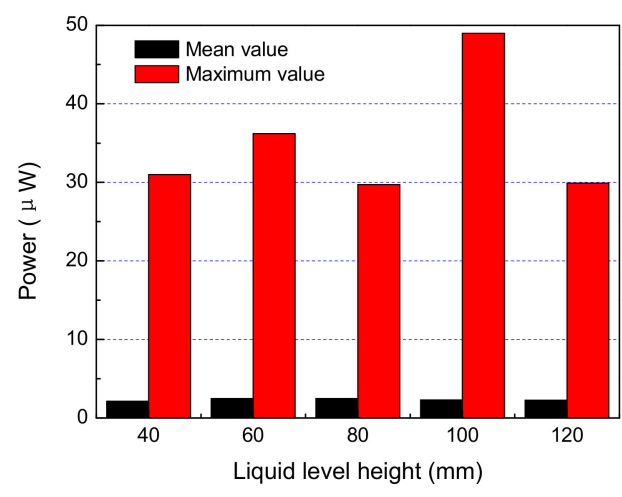

(a)

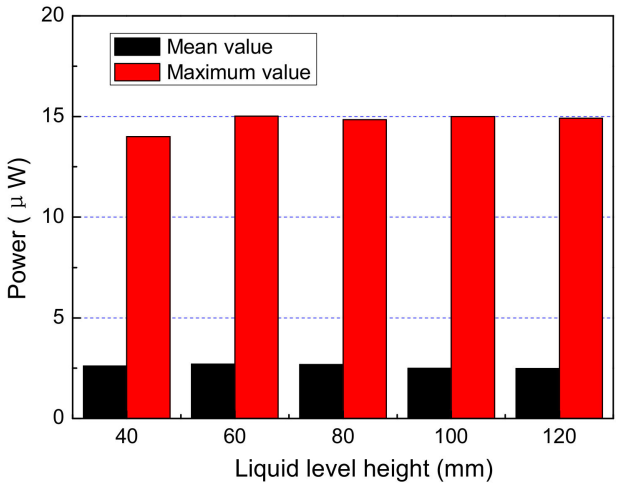

(b)

Figure 7. Bar graph of the hybrid energy harvester output power information: (a) Piezoelectric transducer; (b) Electromagnetic generator.

The output power signal of the piezoelectric transformer changed dramatically. The maximum instantaneous power value was $49.31 \mu \mathrm{W}$ (the liquid level was $100 \mathrm{~mm}$ ). As the liquid level increased from $40 \mathrm{~mm}$ to $120 \mathrm{~mm}$, the average output power of the piezoelectric transformer first increased and then decreased from $2.12 \mu \mathrm{W}$ (the liquid level was $40 \mathrm{~mm}$ ) to $2.47 \mu \mathrm{W}$ (the liquid level was $80 \mathrm{~mm}$ ) and then to $2.24 \mu \mathrm{W}$ (the liquid level was $120 \mathrm{~mm}$ ). When the liquid level was $80 \mathrm{~mm}$, the device reached its optimal average power output state.

Compared with the piezoelectric transformer, the power output of the electromagnetic generator was more stable than violent, and the maximum instantaneous power was $15.02 \mu \mathrm{W}$ (the liquid level was $60 \mathrm{~mm}$ ). When the liquid level increased from $40 \mathrm{~mm}$ to $120 \mathrm{~mm}$, the average output power of the electromagnetic generator first increased and then decreased, from $2.60 \mu \mathrm{W}$ (the liquid level was $40 \mathrm{~mm}$ ) to $2.70 \mu \mathrm{W}$ (the liquid level was $60 \mathrm{~mm}$ ), and then to $2.47 \mu \mathrm{W}$ (the liquid level was $120 \mathrm{~mm}$ ). When the liquid level was $60 \mathrm{~mm}$, the device reached its optimal average power output state.

\subsection{Power Comparison between the Piezoelectric and Electromagnetic Portions}

For the hybrid energy harvester, the piezoelectric transformer was obviously affected by the load conditions; in contrast, the impact on the electromagnetic generator part was small (Figure 8 and Table 3).

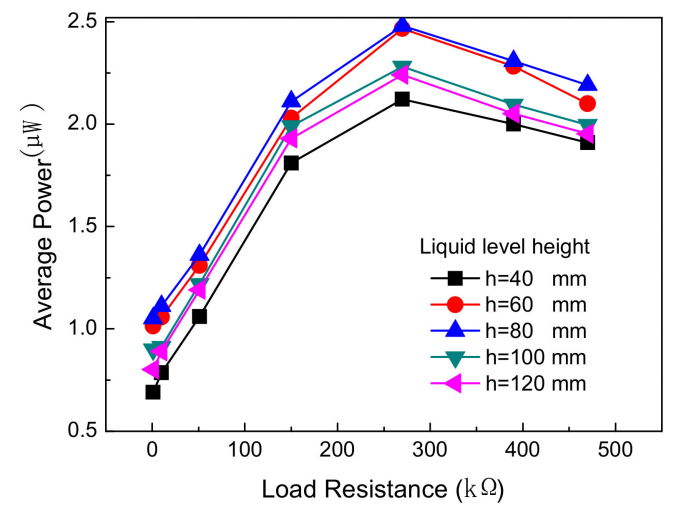

(a)

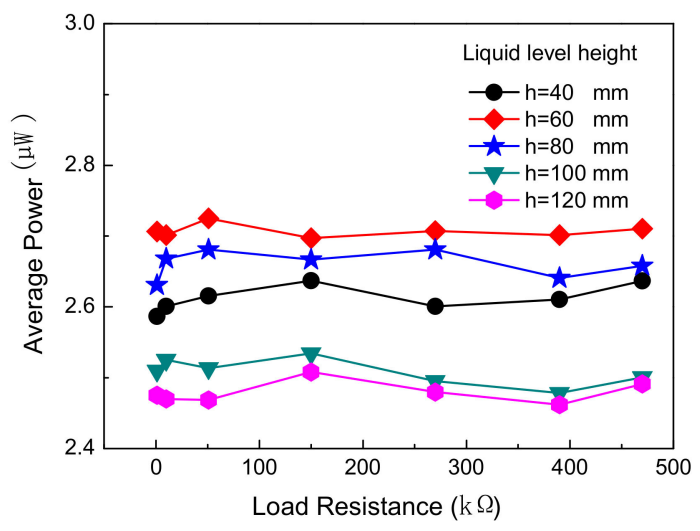

(b)

Figure 8. The influence of circuit load on output power of the hybrid energy harvester: (a) Piezoelectric transducer; (b) Electromagnetic generator. 
As the load resistance of the circuit gradually increased from $1 \mathrm{k} \Omega$ to $470 \mathrm{k} \Omega$, the hybrid energy harvester (the liquid level differed) changed in a consistent manner: first increasing and then decreasing (Figure $7 \mathrm{a}$ and Table 2). The average output power of the hybrid energy harvester was the highest when the resistance value was $270 \mathrm{k} \Omega$ (the maximum value was $2.47 \mu \mathrm{W}$, and the liquid level height was $80 \mathrm{~mm}$; the minimum value was $2.12 \mu \mathrm{W}$, and the corresponding liquid level height was $40 \mathrm{~mm}$ ). The average output power of the device was the lowest when the resistance value is $1 \mathrm{k} \Omega$ (the maximum value was $1.05 \mu \mathrm{W}$, and the liquid level was $80 \mathrm{~mm}$; the minimum value was $0.69 \mu \mathrm{W}$, and the liquid level was $40 \mathrm{~mm}$ ).

\section{Conclusions}

In this study, a hybrid energy harvester for a low-frequency vibration environment in civil engineering was described, including the piezoelectric transformer and electromagnetic generator. The low-frequency sloshing energy collected by this energy harvester can drive microsensors for civil engineering monitoring. The following conclusions can be drawn from the test results.

1. The hybrid energy harvester is especially suitable for the low-frequency vibration environment of civil engineering structure. The natural frequency of the device is within the range of $0.8 \sim 0.93 \mathrm{~Hz}$ when the liquid level is in the range of $40 \sim 120 \mathrm{~mm}$;

2. In the output voltage signal of the hybrid energy harvester, the amplitude of the voltage change of the piezoelectric transformer was far greater than that of the electromagnetic generator. Similarly, the maximum instantaneous output power of the piezoelectric transformer was larger than that of the electromagnetic generator;

3. The power of the piezoelectric transformer of the hybrid energy harvester was clearly affected by the circuit load. The maximum output power was $2.47 \mu \mathrm{W}$ (circuit load $270 \mathrm{k} \Omega$; liquid level height $80 \mathrm{~mm}$ ); the minimum output power was $0.69 \mu \mathrm{W}$ (circuit load $1 \mathrm{k} \Omega$; liquid level height $40 \mathrm{~mm}$ );

4. The influence of circuit load on the electromagnetic generator of the hybrid energy harvester was small. The maximum output power was $2.72 \mu \mathrm{W}$ (circuit load $470 \mathrm{k} \Omega$; liquid level height $60 \mathrm{~mm}$ ), and the minimum output power was $2.45 \mu \mathrm{W}$ (circuit load $1 \mathrm{k} \Omega$; liquid level height $120 \mathrm{~mm}$ ).

Author Contributions: Formal analysis, N.W.; investigation, N.W.; resources, J.F.; data curation, Y.H.; writing-original draft preparation, N.W.; writing—review and editing, P.L. All authors have read and agreed to the published version of the manuscript.

Funding: We thank the National Natural Science Foundation of China (11972123) and the Guangdong Basic and Applied Basic Research Foundation (2019A1515111117) for financial support.

Institutional Review Board Statement: Not applicable.

Informed Consent Statement: Not applicable.

Data Availability Statement: The test raw data used to support the findings of this study are available from the corresponding author upon request.

Conflicts of Interest: We declare that we do not have any commercial or associated interests that might represent a conflict of interest in connection with the work submitted.

\section{References}

1. Jung, S.-M.; Yun, K.-S. Energy-harvesting device with mechanical frequency-up conversion mechanism for increased power efficiency and wideband operation. Appl. Phys. Lett. 2010, 96, 111906. [CrossRef]

2. Khan, F.U.; Iqbal, M. Development of a testing rig for vibration and wind based energy harvesters. J. Eng. Appl. Sci. 2016, 35, 101-110.

3. Gullapalli, H.; Vemuru, V.S.; Kumar, A.; Botello-Mendez, A.; Vajtai, R.; Terrones, M.; Nagarajaiah, S.; Ajayan, P.M. Flexible piezoelectric ZnO-paper nanocomposite strain sensor. Small 2010, 6, 1641-1646. [CrossRef]

4. Liu, L.; Pang, Y.; Yuan, W.; Zhu, Z.; Yang, Y. A self-powered piezoelectric energy harvesting interface circuit with efficiencyenhanced P-SSHI rectifier. J. Semicond. 2018, 39, 4. [CrossRef] 
5. Zhang, Z.; Xiang, H.; Shi, Z.; Zhan, J. Experimental investigation on piezoelectric energy harvesting from vehicle-bridge coupling vibration. Energy Convers. Manag. 2018, 163, 169-179. [CrossRef]

6. Cha, Y.; Chae, W.; Kim, H.; Walcott, H.; Peterson, S.D.; Porfiri, M. Energy harvesting from a piezoelectric biomimetic fish tail. Renew. Energy 2016, 86, 449-458. [CrossRef]

7. Chen, L.; Jiang, W. A piezoelectric energy harvester based on internal resonance. Acta Mech. Sin. 2015, 31, 223-228. [CrossRef]

8. Li, H.; Qin, W. Dynamics and coherence resonance of a laminated piezoelectric beam for energy harvesting. Nonlinear Dyn. 2015, 81, 1751-1757. [CrossRef]

9. Cao, J.; Zhou, S.; Inman, D.J.; Chen, Y. Chaos in the fractionally damped broadband piezoelectric energy generator. Nonlinear Dyn. 2014, 80, 1705-1719. [CrossRef]

10. Galchev, T.; McCullagh, J.; Peterson, R.L.; Najafi, K. A vibration harvesting system for bridge health monitoring applications. Proc. PowerMEMS 2010, 01, 179-182.

11. Galchev, T.V.; McCullagh, J.; Peterson, R.L.; Najafi, K. Energy harvesting of radio frequency and vibration energy to enable wireless sensor monitoring of civil infrastructure. J. Micromech. Microeng. 2011, 21, 1-12.

12. Li, Y.B.; Yao, M.H.; Zhang, W. Experimental study on nonlinear vibration of bistable piezoelectric cantilever beam. Appl. Mech. Mater. 2015, 775, 363-367. [CrossRef]

13. Zhou, S.; Cao, J.; Inman, D.J.; Lin, J.; Liu, S.; Wang, Z. Broadband tristable energy harvester: Modeling and experiment verification. Appl. Energy 2014, 133, 33-39. [CrossRef]

14. Yao, M.H.; Li, Y.B.; Zhang, W. The Steady-State Response Analysis of Bistable Piezoelectric Converter. Appl. Mech. Mater. 2015, 775, 301-306. [CrossRef]

15. Chiu, M.-C.; Karkoub, M.; Her, M.-G. Energy harvesting devices for subsea sensors. Renew. Energy 2017, 101, 1334-1347. [CrossRef]

16. Lin, T.; Pan, Y.; Chen, S.; Zuo, L. Modeling and field testing of an electromagnetic energy harvester for rail tracks with anchorless mounting. Appl. Energy 2018, 213, 219-226. [CrossRef]

17. Gao, M.; Lu, J.; Wang, Y.; Wang, P.; Wang, L. Smart monitoring of underground railway by local energy generation. Undergr. Space 2017, 2, 210-219. [CrossRef]

18. Cahill, P.; Hazra, B.; Karoumi, R.; Mathewson, A.; Pakrashi, V. Vibration energy harvesting based monitoring of an operational bridge undergoing forced vibration and train passage. Mech. Syst. Signal Process. 2018, 106, 265-283. [CrossRef]

19. Alamdari, M.M.; Rakotoarivelo, T.; Khoa, N.L.D. A spectral-based clustering for structural health monitoring of the Sydney Harbour Bridge. Mech. Syst. Signal Process. 2017, 87, 384-400. [CrossRef]

20. Neitzel, F.; Resnik, B.; Weisbrich, S.; Friedrich, A. Vibration monitoring of bridges. Rep. Geodesy 2018, 1, $331-340$.

21. Kwon, S.-D.; Park, J.; Law, K. Electromagnetic energy harvester with repulsively stacked multilayer magnets for low frequency vibrations. Smart Mater. Struct. 2013, 22, 5. [CrossRef]

22. Wang, L.; Yuan, F.G. Vibration energy harvesting by magnetostrictive material. Smart Mater. Struct. 2008, 17, 4.

23. De Marqui, C.; Erturk, A. Electroaeroelastic analysis of airfoil-based wind energy harvesting using piezoelectric transduction and electromagnetic induction. J. Intell. Mater. Syst. Struct. 2012, 24, 846-854. [CrossRef]

24. Wacharasindhu, T.; Li, L.; Kwon, J.W. A micromachined electromagnetic and piezoelectric power harvester from keyboard. J. Micromech. Microeng. 2008. [CrossRef]

25. Challa, V.R.; Prasad, M.G.; Fisher, F.T. A coupled piezoelectric-electromagnetic energy harvesting technique for achieving increased power output through damping matching. Smart Mater. Struct. 2009, 18, 9. [CrossRef]

26. Toyabur, R.M.; Salauddin, M.; Cho, H.; Park, J.Y. A multimodal hybrid energy harvester based on piezoelectric-electromagnetic mechanisms for low-frequency ambient vibrations. Energy Convers. Manag. 2018, 168, 454-466. [CrossRef]

27. Iqbal, M.; Khan, F.U. Hybrid vibration and wind energy harvesting using combined piezoelectric and electromagnetic conversion for bridge health monitoring applications. Energy Convers. Manag. 2018, 172, 611-618. [CrossRef]

28. Huo, L.S.; Li, H.N. Structural vibration control using semi-active variable stiffness tuned liquid column damper. J. Vib. Shock. 2012, 31, 157-164.

29. Sakamoto, D.; Oshima, N.; Fukuda, T. Tuned sloshing damper using electro-rheological fluid. Smart Mater. Struct. 2001, 10, 963-969. [CrossRef] 